\title{
Novel Insights into the Hematological Parameter Abnormalities in Pediatric COVID-19 Cases: Observation from A Preliminary Study of 11 Pediatric COVID-19 Cases in A Tertiary Care Center of North India
}

Dr. Neema Tiwari ${ }^{1}$, Dr. Devajit Nath ${ }^{1 *}$, Dr. Jyotsna Madan ${ }^{1}$, Prashant Bajpai ${ }^{2}$, Dr. Ujjwal Madan ${ }^{3}$, Dr. Savitri Singh ${ }^{1}$

${ }^{1}$ Dept. of Pathology, Superspeciality Pediatric Hospital \& Postgraduate Teaching Institute, Sector 30, Noida-201301, India

${ }^{2}$ Dept. of Biostatistics, University of Lucknow, Lucknow-226025, India

${ }^{3}$ University College of Medical Sciences, New-Delhi -110095, India

DOI: $\underline{10.36348 / \text { sjpm.2020.v05i05.010 }}$ | Received: 29.04 .2020 | Accepted: 15.05 .2020 | Published: 21.05 .2020

*Corresponding author: Dr. Devajit Nath

\section{Abstract}

Introduction: The Corona Virus Disease 2019, caused by novel Coronavirus SARS-CoV-2 is an acute respiratory and systemic illness, and is declared as a global pandemic by WHO on the $11^{\text {th }}$ of March 2020.0 nly $2.2 \%$ to $1.7 \%$ of pediatric COVID-19 cases have been reported from USA and China respectively. The hematological parameter alteration in the pediatric COVID-19 cases needs to be addressed in the Indian population. Aim: To assess the alteration of the baseline hematology parameters in the paediatric COVID-19 cases and to compare the values with the adult COVID-19 cases for any statistically significant difference. Methods: A single Centre retrospective study of 11 COVID-19 asymptomatic pediatric patients and asymptomatic gender-matched adult cases admitted to SSPH\&PGTI NOIDA from March to April, were enrolled for the study. The demographic date, baseline hematological findings of both groups were recorded and analyzed statistically. Results: Demographic characterization shows a mean age of 8.7 years in the pediatric population. Baseline CBC findings of all the pediatric cases show mild anemia, normal leucocyte, normal absolute lymphocyte count, and neutrophilia.- Majority of the pediatric cases have NLR value below the cut-off point of 3.13.- PLR was slightly higher in pediatric cases. Comparison between two groups shows statistically significant differences for the hemoglobin and platelet value only. Conclusion: The comparative analysis of baseline hematologic parameters did not show any significant derangement of the CBC parameters in pediatric cases. Significance in the difference of Hemoglobin level and platelet count is observed in comparison to the adult cases.

Key words: Corona virus, COVID-19, Pediatric, CBC, NLR, PLR.

Copyright @ 2020: This is an open-access article distributed under the terms of the Creative Commons Attribution license which permits unrestricted use, distribution, and reproduction in any medium for non-commercial use (NonCommercial, or CC-BY-NC) provided the original author and sources are credited.

\section{INTRODUCTION}

The Corona Virus disease (COVID-19) is an acute respiratory and systemic illness, caused by novel Coronavirus SARS-CoV-2 and is declared as a global pandemic by WHO on $11^{\text {th }}$ March 2020. Humans of all ages are susceptible to the virus. However, as compared to adults, the number of reported Covid-19 cases in the pediatric population is low. Globally the Chinese literature has reported a percentage of $2.2 \%$ of Pediatric COVID-19 out of 44,672 confirmed cases as on $11^{\text {th }}$ February 2020[1]. The United States has reported a percentage of $1.7 \%$ of Pediatric COVID-19 out of 149082 confirmed cases between $12^{\text {th }}$ February and $02^{\text {nd }}$ April 2020[2]. Italy and Spain reported a 1.2\% [3] and $0.8 \%$ [4] of confirmed pediatric COVID-19 Cases, respectively[5].
As the pandemic is progressing over time, India has reported over 56000 cases so far with as many as 1886 death as of $8^{\text {th }}$ May 2020 as per Ministry of Health and Family Welfare Government of India (MOHFWGOI) website data, however, there is a paucity of data with limited information on the incidence of pediatric COVID-19 in the Indian population.

Various hematological parameters alteration has been documented in the current literature review in SARS-Cov-2 infection primarily from the adult cases in the Chinese and the Western population, which signifies the severity and the prognosis of the patient outcome. As per the Diagnosis and Treatment Guidelines (trial version 7) [6] review, in the early stage of the disease onset, total leukocyte count is normal, and the lymphocyte count is reduced. From a study 
conducted by Li et al. [7] the lymphocyte percentage (Lym\%) was found to be inversely related to the severity and prognosis of patients, which in turn could be used to predict the severity and prognosis of patients with COVID-19. The hematological alteration has the potentiality to serve as indicators to provide guidance for the diagnosis, treatment, and prognosis for COVID19 patients. The documentation of the hematological parameter alteration is very limited in the pediatric COVID-19 cases worldwide and in India as a whole owing to the relatively lower incidence of the disease in the pediatric population.

The present study was undertaken with the aim to assess the characteristic changes in the value of hematology routine parameters in the pediatric COVID19 cases in the Indian scenario. In order to explore the value of hematology routine parameters, we retrospectively analyzed the baseline hematology test results of 11 asymptomatic pediatric COVID-19 cases. The secondary objective of the study was to compare the baseline hematology parameters of the pediatric cases with the adult COVID-19 cases to look for any statistically significant difference between the two groups.

\section{METHOD \\ Patient selection}

A single-center retrospective study of 11 pediatric patients with laboratory-confirmed COVID-19 admitted to Super Speciality Pediatric Hospital \& Post Graduate Teaching Institute(SSPH\&PGTI), NOIDA from March to April, were enrolled for the study. The 11 pediatric cases were asymptomatic on the day of admission. For comparative analysis, gender-matched 11 asymptomatic laboratories confirmed adult cases were also enrolled for the study. The diagnosis of COVID-19 was according to the Ministry of Health and Family Welfare (MOHFW) Government of India (GOI) guidelines and confirmed by RTPCR performed on nasopharyngeal and throat swab samples of the patient. Patients with preexisting hematological or underlying disorders were excluded in both the pediatric and the adult group.

\section{Baseline data Collection}

The demographic data and hematological findings of the pediatric and the adult group were recorded. The samples for complete blood count (CBC) for both the group were collected on the day of admission. All patients didn't receive any treatment before blood sampling. The samples were tested for complete blood count on 5 part hematology analyzer Abbott Cell Dyn Ruby, and values were noted. The hematology test results were divided into two groups based on the sources of the samples as pediatric and adult on which retrospective and comparative analysis was performed.

\section{STATISTICAL ANALYSIS}

Statistical analysis was performed with the Statistical Package for the Social Sciences (SPSS version 15.0, SPSS Inc., Chicago, IL, USA) software. Normally distributed continuous variables were presented as mean, median, Standard deviation (SD), Minimum, maximum, and $95 \%$ C.I (Confidence Interval). The respective hematology parameters of the two groups were compared using two-sample t-Test at a $5 \%$ level of significance.

\section{RESULTS \\ Demographic characteristics}

The mean age of presentation in adult cases is 37.3 years, while in pediatric cases, the mean age is 8.7 years. The youngest case we had was of 4 months of age. Each cohort had 4(36.3\%) females and 7 males $(63.6 \%)$ out of 11 cases (Fig-1).

\section{Hematology findings}

Baseline hematology finding in the pediatric cases and the comparative analysis with the adult cases are presented in Table 1 and Table 2. The boxplots for these parameters are presented in Figure 2-11(Legend: Composite analysis of variables under study for baseline values between the pediatric and the adult group (box and whisker plots) show range of variation in different parameters. The lines show variation beyond the upper and lower ranges with each plot with random outliers. Each plot has a central line showing the range of dispersion of values and in the composite plots there is variation in the value of Hemoglobin and the platelet count.)

Anemia was found in $54.5 \%$ (6/11) of children while in $45.5 \%(5 / 11)$ cases have a hemoglobin level less than $10 \mathrm{mg} / \mathrm{dl}$. $18.2 \%(2 / 11)$ of adult cases have anemia, while only 1 case $(9.1 \%)$ has a hemoglobin level of less than $10 \mathrm{mg} / \mathrm{dl}$. (Figure2). Hemoglobin level is higher in Adult patients as compared to pediatric patients, and there is a statistically significant difference $(\mathrm{p}<0.05)$ in the Hemoglobin between Pediatric \& Adult patients (Table 2). RBC count is higher in Adult patients as compared to pediatric patients, but there is no statistically significant difference in the RBC count between the Pediatric \& the Adult patients. (Figure 3, Table 2).

WBC count is higher in Pediatric patients as compared to Adult patients, but there is no statistically significant difference in the WBC count between Pediatric \& Adult patients (Figure 4, Table 3). Neutrophilia is observed in $36.3 \%$ (4/11) cases and children younger than 10 years, showing more predominant neutrophilia as compared to children above 10 years. In adult cases,27.3\%(3/11) showed neutrophilia. The neutrophil count is higher in Pediatric patients as compared to Adult patients, but there is no statistically significant difference in the Neutrophil count between the Pediatric \& the Adult patients 
(Figure 5, Table 2).

Of the pediatric cases, $27.3 \%$ (3/11) showed lymphopenia, $27.3 \% \quad(3 / 11) \quad$ cases showed lymphocytosis, while only $9.1 \%(1 / 11)$ of the adult cases showed lymphopenia. Lymphocyte count is slightly higher in Pediatric patients as compared to Adult patients, but there is no statistically significant difference in the Lymphocyte count between Pediatric \& Adult patients (Figure 6, Table 2).

Monocyte count was reduced to the lower normal limit in $72.7 \%(8 / 11)$ of the pediatric cases, with $18.2 \%(2 / 11)$ cases showed monocytosis- Mild monocytosis is observed in only $1(9.1 \%)$ adult case. Monocyte count is slightly lower in Pediatric patients as compared to Adult patients, but there is no statistically significant difference in the Monocyte count between Pediatric and adult patients (Figure 7, Table 2).

Eosinophil count is slightly lower in Pediatric patients, while in adults, $27.3 \%$ (3/11) cases showed Eosinophilia with remaining values were within the normal range, but there is no statistically significant difference in the Eosinophils count between Pediatric \& Adult patients ( Figure 8, Table 2).

Platelet count is higher in Pediatric patients as compared to adult patients, and there is a statistically significant difference in the Platelet count between Pediatric \& Adult patients $(\mathrm{p}<0.05) .18 .2 \% \quad(2 / 11)$ pediatric cases showed some degree of thrombocytopenia, while $36.3 \%(4 / 11)$ of adult cases had thrombocytopenia(Figure 9, Table 2).

The Neutrophil-Lymphocyte Ratio (NLR) is taken as a cut-off of 3.13 as a low-risk group stratification for age less than 50 years. The neutrophillymphocyte ratio (NLR) in the pediatric cases showed that $27.3 \%(3 / 11)$ cases had raised NLR above the cut off value of 3.13 . NLR is slightly higher in Pediatric patients as compared to Adult patients, but there is no statistically significant difference in the NLR between Pediatric \& Adult patients (Figure 10, Table 2). PLR is slightly higher in Pediatric patients as compared to Adult patients, but there is no statistically significant difference in the PLR between Pediatric \& Adult patients (Figure 11, Table 2).

Table-1: Basic statistical values for different variables in the Paediatric and the adult population

\begin{tabular}{|l|l|l|l|}
\hline \multicolumn{5}{|c|}{ PEDIATRIC CASES } \\
\hline Variables & Mean & Median & $\begin{array}{l}\text { Standard } \\
\text { deviation }\end{array}$ \\
\hline RBC & 4.4 & 4.5 & 0.8 \\
\hline Hb & 10.6 & 10.9 & 1.8 \\
\hline WBC & 11.5 & 11.0 & 5.9 \\
\hline Neutrophil & 7.2 & 6.4 & 6.2 \\
\hline Lymphocyte & 3.0 & 2.1 & 2.3 \\
\hline Monocyte & .42 & .39 & .34 \\
\hline eosinophil & 0.13 & 0.06 & 0.15 \\
\hline platelet & 230.0 & 245.0 & 102.6 \\
\hline NLR & 4.1 & 1.8 & 5.25 \\
\hline PLR & 109.31 & 68.3 & 49.7 \\
\hline \multicolumn{5}{|c|}{ ADULT CASES } \\
\hline Variables & Mean & Median & Standard \\
& & & deviation \\
\hline RBC & 4.4 & 4.8 & 0.91 \\
\hline Hb & 12.7 & 13.3 & 2.4 \\
\hline WBC & 7.9 & 6.1 & 4.5 \\
\hline Neutrophil & 4.9 & 3.4 & 3.9 \\
\hline Lymphocyte & 2.1 & 1.9 & .76 \\
\hline Monocyte & .45 & .37 & .42 \\
\hline eosinophil & 0.31 & 0.13 & 0.36 \\
\hline platelet & 129.0 & 125.0 & 97.1 \\
\hline NLR & 2.7 & 1.5 & 2.68 \\
\hline PLR & 71.52 & 58.5 & 87.5 \\
\hline
\end{tabular}

Table-2: T-test for comparison of onetime baseline haematological parameters of 11 paediatric and the adult cases on the day of admission

\begin{tabular}{|c|c|c|c|c|c|c|c|}
\hline \multirow{3}{*}{$\begin{array}{l}\text { Pediatric Vs } \\
\text { Adult }\end{array}$} & \multicolumn{7}{|c|}{ t-test for Equality of Means } \\
\hline & \multirow[t]{2}{*}{$\mathbf{t}$} & \multirow[t]{2}{*}{ df } & \multirow[t]{2}{*}{$\begin{array}{c}\text { p- } \\
\text { value }\end{array}$} & \multirow[t]{2}{*}{$\begin{array}{c}\text { Mean } \\
\text { Difference }\end{array}$} & \multirow[t]{2}{*}{$\begin{array}{l}\text { Std. Error } \\
\text { Difference }\end{array}$} & \multicolumn{2}{|c|}{$\begin{array}{l}\text { 95\% Confidence Interval } \\
\text { of the Difference }\end{array}$} \\
\hline & & & & & & Lower & Upper \\
\hline $\mathrm{RBC}$ & -.059 & 20 & 0.953 & -.02182 & .36744 & -0.79 & 0.75 \\
\hline $\mathrm{HB}$ & -2.271 & 20 & 0.034 & -2.13545 & .94015 & -4.09 & -0.17 \\
\hline WBC & 1.375 & 20 & 0.184 & 3.08273 & 2.24188 & -1.59 & 7.76 \\
\hline Neutrophil & 1.046 & 20 & 0.308 & 2.33182 & 2.22832 & -2.32 & 6.98 \\
\hline Lymphocyte & 1.354 & 20 & 0.191 & .991545 & .732422 & -.536 & 2.52 \\
\hline Monocyte & -.197 & 20 & 0.846 & -.032636 & .165785 & -0.38 & 0.32 \\
\hline Eosinophils & -1.502 & 20 & 0.149 & -.178364 & .118714 & -0.43 & 0.07 \\
\hline Platelet & 2.372 & 20 & 0.028 & 101.07091 & 42.60493 & 12.19 & 189.94 \\
\hline NLR & .767 & 20 & 0.452 & 1.36455 & 1.77981 & -2.35 & 5.08 \\
\hline PLR & 1.245 & 20 & 0.228 & 37.78909 & 30.35321 & -25.53 & 101.11 \\
\hline
\end{tabular}




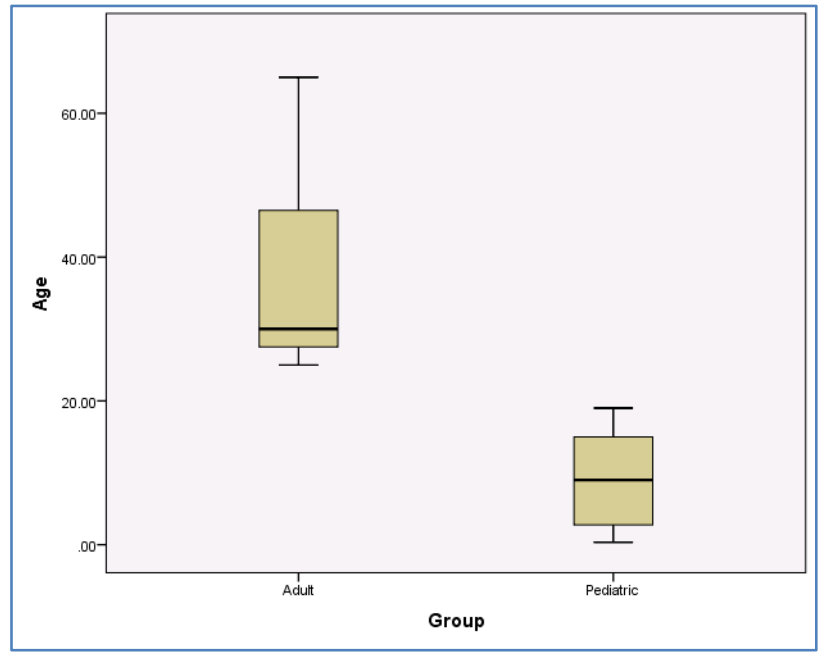

Fig-1

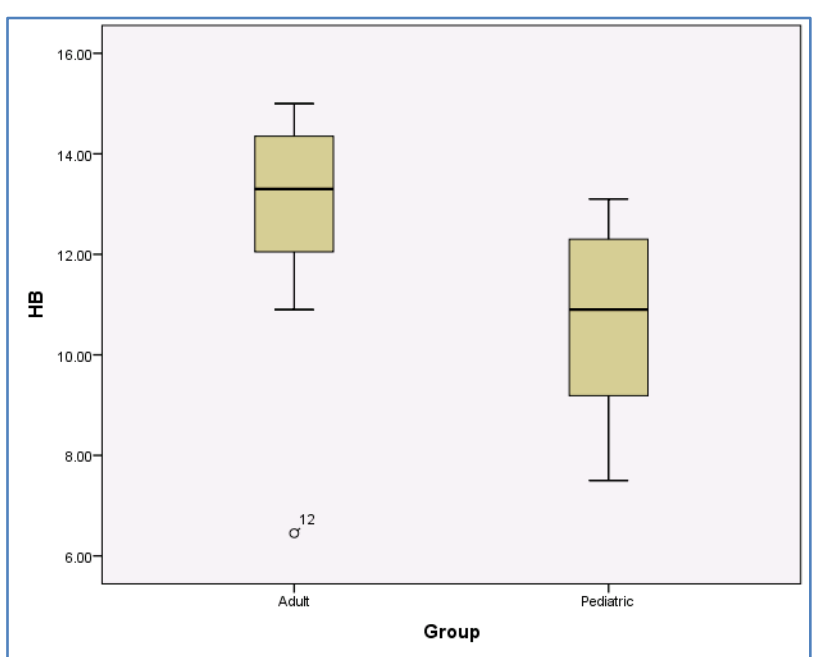

Fig-2: Hemoglobin

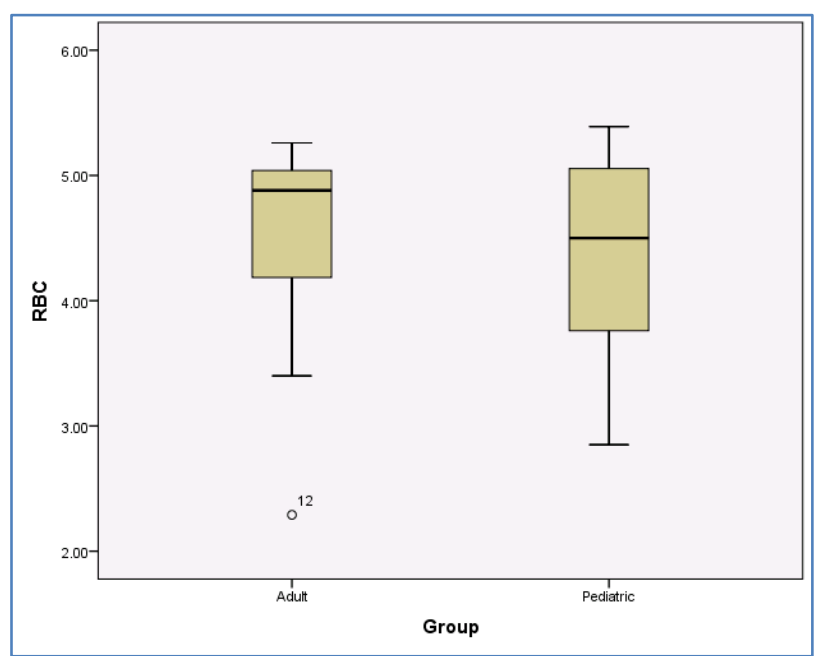

Fig-3: RBC Count

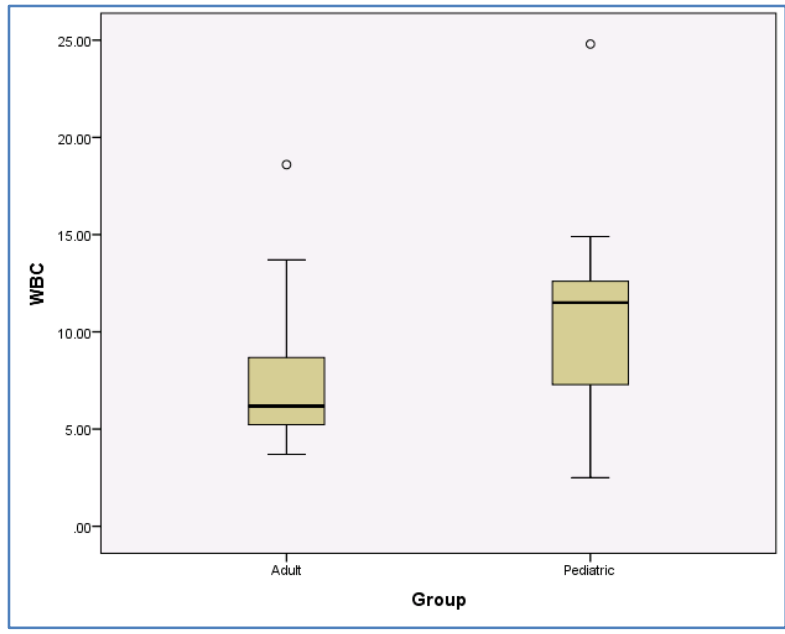

Fig-4: WBC count

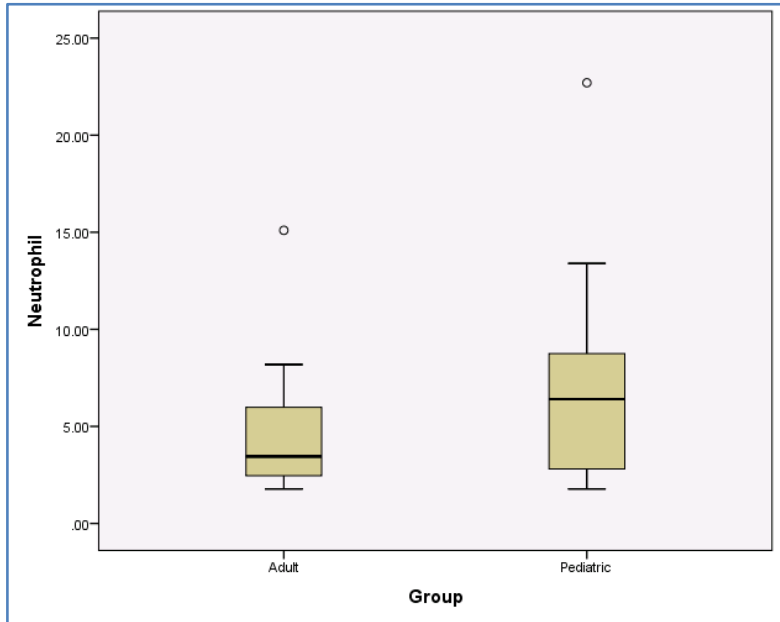

Fig-5: Absolute Neutrophil Count

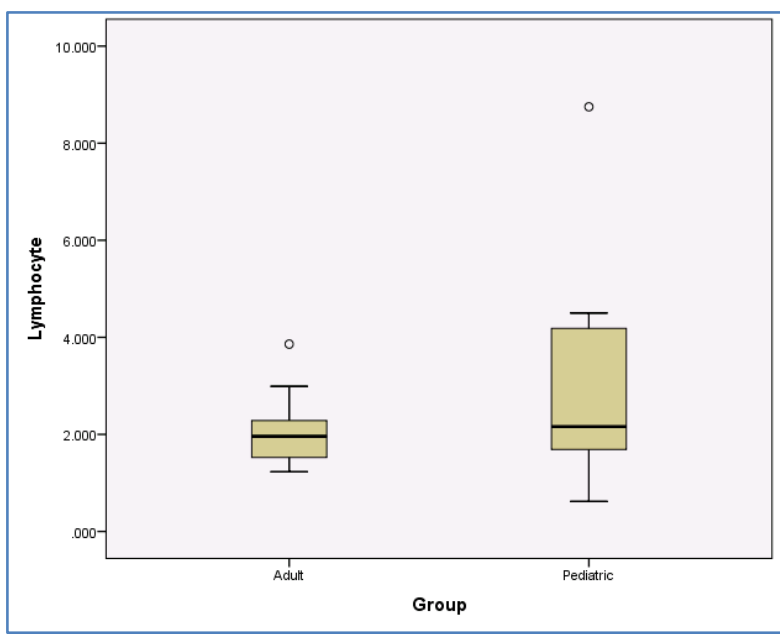

Fig-6: Absolute Lymphocyte Count 


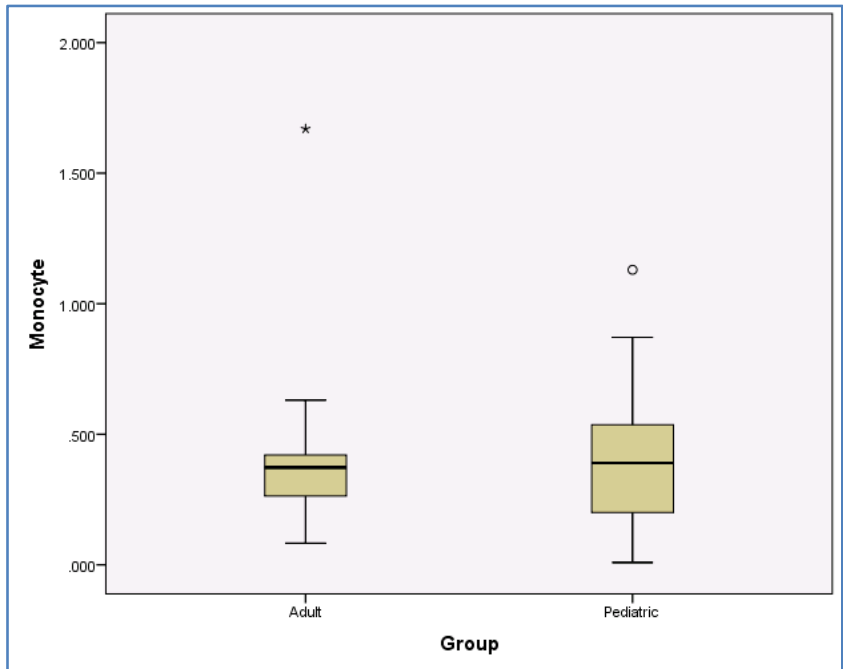

Fig-7: Absolute Monocyte Count

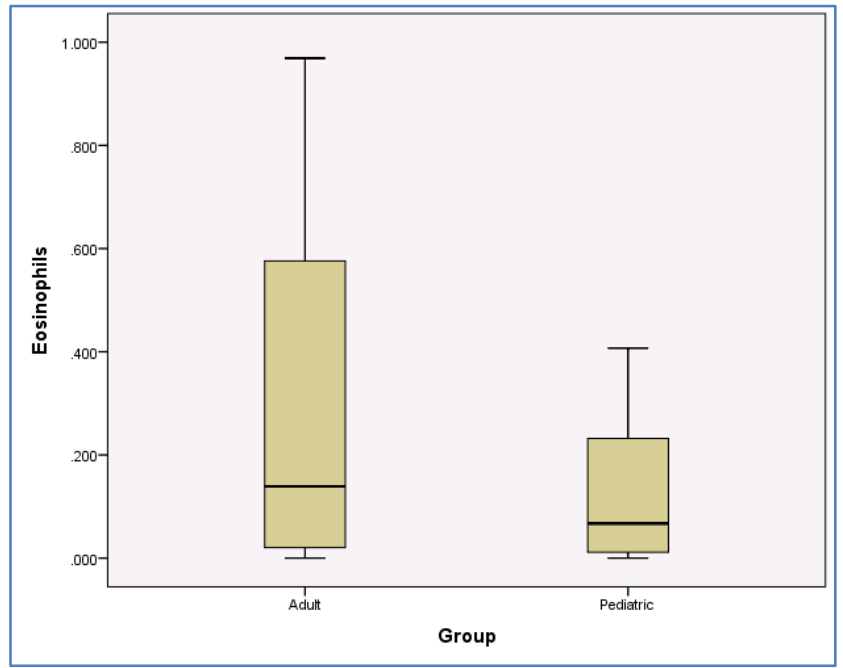

Fig-8: Absolute Eosinophil Count

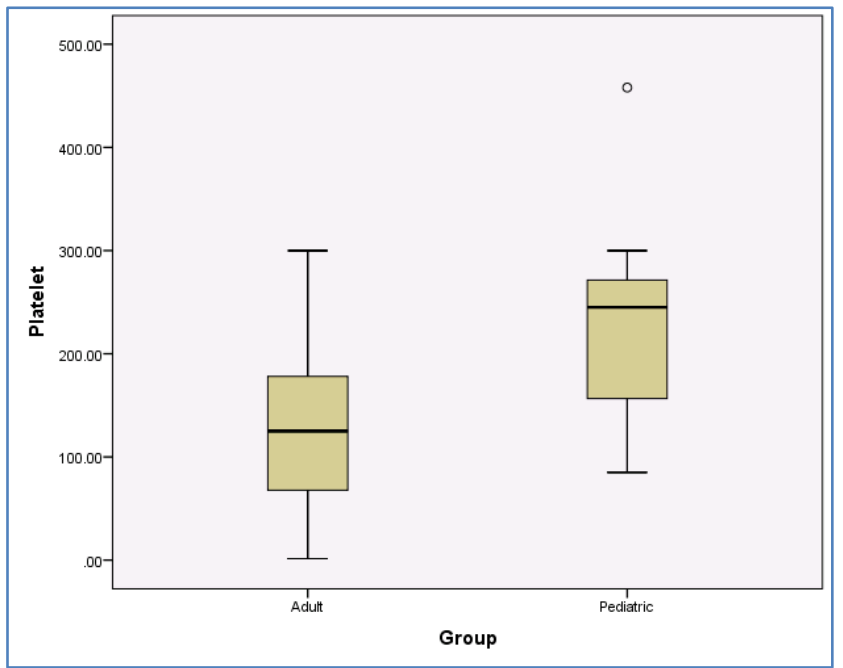

Fig-9: Platelet Count

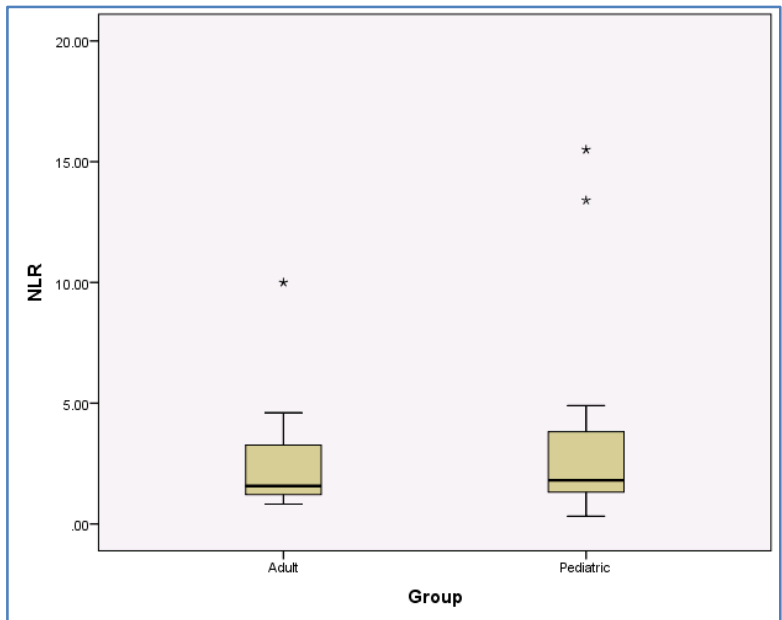

Fig-10: NLR

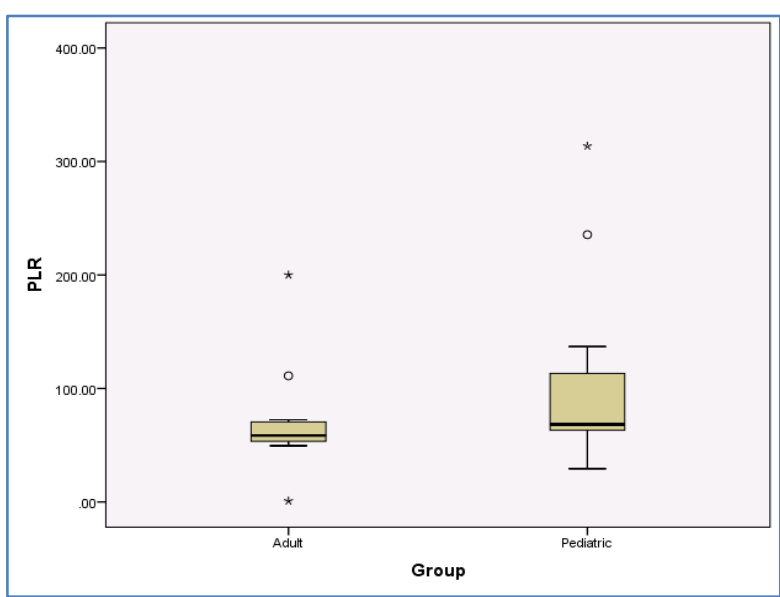

Fig-11: PLR

Fig.1-11(Legend: Composite analysis of variables under study for baseline values between the pediatric and the adult group (box and whisker plots) show range of variation in different parameters. The lines show variation beyond the upper and lower ranges with each plot with random outliers. Each plot has a central line showing the range of dispersion of values and in the composite plots there is variation in the value of Hemoglobin and the platelet count.)

\section{DISCUSSION}

Coronavirus disease caused by coronavirus SARS-CoV-2 belongs to the $\beta$-coronavirus cluster and is the third known cause of zoonotic coronavirus disease after severe acute respiratory syndrome (SARS) and the Middle East respiratory syndrome (MERS) ${ }^{8}$ Early reports from Wuhan, China showed that all of the COVID-19 patients were middle-aged and elderly ( http://2019ncov.chinacdc.cn as accessed on $10^{\text {th }}$ of May 2020)[8]. As the disease enters into the epidemic stage, pediatric patients (even newborns) have been reported increasingly $[9,10]$. According to the latest report from the Chinese Center for Disease Control and Prevention (CCDC), 965 confirmed patients below 19 years $(2.16 \%, 965 / 44,672)$ were reported nationwide[1]. 
The relatively lower percentage of cases in the pediatric population as compared to adults follows a similar epidemiological pattern that was observed in the SARS and MERS outbreak [11]. Chinese literature suggest that median age of pediatric cases is 6.7 years(Range 1day-15 years) with clinically pediatric COVID-19 cases might be less severe than cases in adults and the children might experience different symptoms than do adults[12,13].

Demographic data from other studies document that most of the patient's age ranged from 2 weeks to 17 years, and females accounted for $54.5 \%$ of cases[11].

In our study, the patients are asymptomatic, and the age ranged from 4 months to 19 years (median age 9.0 years), and most were males (64\%). Males seemed to be more susceptible to SARS-CoV-2 infection, which was similar to previously reported study[14-17].

Of the hematological parameters, lymphopenia had been the most common documented findings on complete blood count panel for adult patients with COVID-19 infection[14,18], whereas this finding has not been seen consistently in the pediatric population. Majority of the pediatric study showed normal white blood cell counts with lymphopenia ranging from $35 \%$ patients in a case series of 20 patients [19] to $3.5 \%$ in a study describing 171 children[19].

Wang et al. [19], in their study, documented that $3.0 \%$ of infants presented with lymphopenia, which included only patients with mild, moderate, or asymptomatic disease. The lack of significant lymphopenia may, in part, explain the limited number of severe COVID-19 in children. Yang et al. [25] reported that $80 \%$ of critically ill adult COVID-19 patients had lymphopenia, whereas Chen et al. [20] reported that only $25 \%$ of patients with mild COVID-19 had lymphopenia, suggesting that lymphopenia may correlate with the severity of the infection. In both SARS and MERS, lymphopenia was a predominant feature, due to a combination of viral particle-induced cytoplasmic damage and apoptosis [21-23]. In very young children; however, lymphopenia may not occur due to the relative immaturity of their immune system and differences in their immune response compared to adults [24]. Reports from some previous studies indicated a decreased lymphocytes count in severe cases of infection $[14,20]$. As a result of viral infection, white blood cell and lymphocyte counts can be reduced by consumption.

Results from our study showed that $27.3 \%$ $(3 / 11)$ of the pediatric cases had lymphopenia, $27.3 \%(3 / 11)$ cases had lymphocytosis, and 45.5\%(5/11) cases had normal absolute lymphocyte count while only 9.1\%(1/11) of the adult cases showed lymphopenia. Lymphocyte count was slightly higher in Pediatric patients as compared to Adult patients, but there was no statistically significant difference in the Lymphocyte count between the Pediatric \& the Adult patients. The finding of lymphopenia in our study is correlating with various previous studies conducted elsewhere; however, the finding of lymphocytosis in the pediatric cases needs further research to document the pathogenesis.

The total leucocyte count did not show any consistent derangements in the various studies conducted earlier in adult cases. A normal leukocyte count was found in $69.6 \%$, with leucocytosis in $15.2 \%$ and leucopenia in another $15.2 \%$ relative to the locally defined reference limits [13]. In our study, a normal total leukocyte count was found in $36.3 \%(4 / 11)$, leucopenia in $9.1 \%(1 / 11)$, and leucocytosis in another $54.5 \%(6 / 11)$ of cases. WBC count is higher in Pediatric patients as compared to Adult patients, but there is no statistically significant difference in the WBC count between Pediatric \& Adult patients.

The neutrophil count from the earlier pediatric studies showed that $89.4 \%$ cases had normal neutrophil count, $4.6 \%$ of cases had neutrophilia, and $6.0 \%$ cases had neutropenia. These findings were in contrast to adult study whereby increased leucocyte and neutrophil counts were common in patients with unfavorable COVID-19 progression[25].

In another study by Cai et al. [26] in 10 symptomatic children of COVID 19 infection with radiological features of pneumonia, only one of the three cases had alterations in leucocyte and neutrophil counts. In our study, Neutrophilia is observed in $36.3 \%$ cases and children younger than 10 years showing more predominant neutrophilia as compared to children above 10 years. In adult cases,27.3\% showed neutrophilia. The neutrophil count is higher in Pediatric patients as compared to Adult patients, but there is no statistically significant difference in the Neutrophil count between the Pediatric \& the Adult patients.

A study conducted on adult cases from China highlights eosinopenia as a significant prognosticating factor [27]. The study proposes Eosinopenia as a potentially more reliable laboratory predictor of SARSCoV-2 infection than recommended "leukocyte counts" and "lymphopenia[27]".

In our study, Eosinophil count is slightly lesser in Pediatric patients, while $27.3 \%$ of the adult cases showed Eosinophilia. Eosinopenia was seen in $36.3 \%$ (4/11) of the pediatric cases and $27.3 \%$ (3/11) of the adult cases, but there was no statistically significant difference in the Eosinophils count between Pediatric \& Adult patients. No consistent eosinophil count abnormality is observed in our study for pediatric cases, unlike the adults where eosinopenia has been described in the studies. 
In the present study, monocyte count was normal in $81.8 \%$ of the pediatric cases, while only $18.2 \%$ cases showed mild monocytosis, whereas mild monocytosis is noted in $9.1 \%$ of the adult cases. The finding of monocytosis in the minority of the pediatric cases has not been documented in other studies.

The platelet count from the pediatric cases is within the normal limit in $81.8 \%$ of cases, while $18.2 \%$ of cases showed some degree of thrombocytopenia, while $36.3 \%$ of adult cases had thrombocytopenia. Platelet count is higher in Pediatric patients (mean $=2.3 \mathrm{~L})$ as compared to Adult patients (mean $=1.29 \mathrm{~L})$, and there is a statistically significant difference in the Platelet count between Pediatric \& Adult patients.

Findings from a study of 30 adult COVID-19 cases conducted in Huizhou, China showed that the patients with markedly elevated platelets and longer average hospitalization days might be related to the cytokine storm.

The Platelet Lymphocyte Ratio (PLR) is a parameter for the degree of cytokine storm, which might provide a new indicator in the monitoring of patients with COVID-19 [27,28].

The PLR in our study was slightly higher in the Pediatric patients (mean=109.31) as compared to Adult patients (mean=71.52), but there is no statistically significant difference in the PLR between Pediatric \& Adult patients.

The Neutrophil-to-Lymphocyte ratio (NLR) is a biomarker for the assessment of the severity of bacterial infections and the prognosis of patients with pneumonia and tumor. NLR may serve as a surrogate marker of early risk identification in COVID 19 infection. In a study by Forget et al. [29] identified that normal NLR values in an adult, non-geriatric population in good health are between 0.78 and 3.53. Whereas, in another study by Jingyuan Li [30], it was concluded that Patients with age $\geq 50$ having NRL $\geq 3.13$ are at risk of severe illness, and they should get rapid access to the intensive care unit, if necessary.

The neutrophil-lymphocyte ratio(NLR) in the pediatric cases showed that $27.3 \%$ of cases had raised NLR above the cut off value of 3.13. The mean NLR of the adult cases was 2.7 below the cut-off value of 3.13.NLR is slightly higher in Pediatric patients(Mean=4.1) as compared to Adult patients(Mean=2.7), but there is no statistically significant difference in the NLR value between Pediatric \& Adult patients.

In a recently discussed paper, it is suggested that in addition to various mechanisms, SARS-CoV-2 viral protein infects hemoglobin by the immune hemolysis of red blood cells[31].

In our study, $54.5 \%$ of children have anemia, with $45.5 \%$ cases have hemoglobin level less than $10 \mathrm{mg} / \mathrm{dl}$, while only $18.2 \%$ of adult cases have anemia with only 1 case $(9.1 \%)$ has hemoglobin level less than $10 \mathrm{mg} / \mathrm{dl}$. Hemoglobin level is higher in adult patients as compared to pediatric patients, and there is a statistically significant difference $(p \leq 0.05)$ in the Hemoglobin between Pediatric \& Adult patients. RBC count is higher in Adult patients as compared to pediatric patients, but there is no statistically significant difference in the RBC count between the Pediatric \& the Adult patients.

\section{CONCLUSION}

The study of the 11 pediatric asymptomatic COVID-19 cases of the Indian population shows an age range of 4 months to 19 years with a male predominance.

- Baseline CBC findings of all the pediatric cases show mild anemia, normal leucocyte, and absolute lymphocyte count in the majority of the cases and neutrophilia in a minority of the cases. The normal count of Eosinophil, monocyte, and platelet, were noted.

- Majority of the pediatric cases had a NLR below the cut-off point of 3.13 .

- PLR was slightly higher in pediatric cases.

- The comparative analysis of the baseline CBC parameters between the pediatric and the adult cases show statistically significant differences for the hemoglobin and for the platelet value while all other parameters did not show any statistical difference between the two groups.

\section{SUMMARY}

Results from the baseline hematologic parameters of pediatric COVID-19 cases and its comparative analysis with the adult cases did not show any significant derangement of the CBC parameters in the pediatric COVID-19 cases in the Indian scenario. Significance in the difference of Hemoglobin level and platelet count is observed in comparison to the adult cases. In summary, unlike in adults, a consistent pattern of hematology parameter alteration has yet to be observed in children with confirmed COVID-19. The laboratory alternations reported in children with SARSCov-2 infections worldwide are not consistent with the early observations in cases of COVID-19 infection in India.

\section{LIMITATION OF THE STUDY}

There are some limitations to our findings. Only 11 asymptomatic pediatric cases were included. Moreover, laboratory methods and reference ranges may have differed between centers and were not always 
clearly defined. Importantly, a lack of linearity of data prevents comparison of laboratory values between symptomatic and asymptomatic children or analysis based on the severity of illness. The cut-off value of NLR for risk stratification with age $(\geq 50,<50)$ is set at 3.13; however, a standard cut-off value for a pediatric age group may have to be defined with a large cohort study. A lack of follow up CBC Parameters in the study group is also a limitation for statistical inference.

\section{RESEARCH IN CONTEXT \\ Special considerations for pediatrics}

Importantly, unlike adults, the laboratory profile in asymptomatic to severe COVID-19 pediatric patients is not clear and does not appear to be consistent with earlier infection like in SARS. Recent guidelines recommend clinicians to monitor lymphocyte and neutrophil count as a prognostic indicator to assess the severity of the infection. A preliminary description of pediatric U.S. COVID-19 cases was published by the CDC on April 10th, 2020. In line with data from China, the CDC reports that pediatric COVID-19 cases might be less severe when compared to adults and that children may experience different symptoms Additionally, although most cases reported among children to date have not been severe, the CDC recommends clinicians maintain a high index of suspicion for COVID-19 infection in children and monitor for progression of illness, particularly among infants and children with underlying conditions. Importantly, interpretation of pediatric laboratory tests based on known adult trends may not be recommended due to lack of pediatric information and characteristics and need more research in the area.

\section{Known facts about the topic}

Sparse literature is available on the hematology laboratory parameter alteration among the pediatric covid-19 cases in the Indian population, where the pandemic is spreading with gradual and greater involvement of the pediatric population.

\section{Added information from the study}

The study obtained data from 11 pediatric COVID-19 cases in NOIDA, India, to explore the epidemiology and the baseline hematological parameters of the patients. The pilot study provides further information on the comparative hematological parameters of pediatric patients with adult cases, thereby adding substantial inputs to the knowledge database.

\section{Conflict of Interest}

All authors declare no competing interests.

\section{Author contribution}

The authors of the manuscript declare an equal contribution to the study.

\section{Ethics committee approval}

No Patient identifiers were collected, and approval of exemption for informed consent was applied.

We thank the Department of Pediatric Medicine, SSPHPGTI, NOIDA, India, and the Internal Medicine, Government District Hospital, NOIDA, India for their support in carrying out the study and the technical staff, Department of Pathology, SSPHPGTI, NOIDA for their effort in carrying out the laboratory investigations. Special acknowledgment to Jahnu Saikia, Ph.D. Scholar, Department of Biosciences and Bioengineering, Indian Institute of Technology Guwahati, India, for his contribution in supervising the final proofreading and the references of the manuscript. We also greatly appreciate the efforts of healthcare workers and the support of their families during this outbreak.

\section{REFERENCES}

1. Wu, Z., \& McGoogan, J. M. (2020). Characteristics of and important lessons from the coronavirus disease 2019 (COVID-19) outbreak in China: summary of a report of 72314 cases from the Chinese Center for Disease Control and Prevention, Jama 323, 1239-1242.

2. Covid, C., COVID, C., COVID, C., Bialek, S., Gierke, R., Hughes, M., McNamara, L. A., Pilishvili, T., and Skoff, T. (2020). Coronavirus Disease 2019 in Children-United States, February 12-April 2, 2020, Morbidity and Mortality Weekly Report 69, 422.

3. Livingston, E., \& Bucher, K. (2020). Coronavirus disease 2019 (COVID-19) in Italy, Jama 323, 1335-1335.

4. Tagarro, A., Epalza, C., Santos, M., SanzSantaeufemia, F. J., Otheo, E., Moraleda, C., and Calvo, C. (2020). Screening and severity of coronavirus disease 2019 (COVID-19) in children in Madrid, Spain, JAMA pediatrics.

5. McIntosh, K., Hirsch, M. S., \& Bloom, A. (2020). Coronavirus disease 2019 (COVID-19), 2020]. Disponível em: https://www. uptodate. com/contents/coronavirus-disease-2019-covid-19epidemiology-virology-clinical-features-diagnosisand-prevention. Acesso em 30.

6. Zhao, J.-Y., Yan, J.-Y., \& Qu, J.-M. (2020) Interpretations of "Diagnosis and Treatment Protocol for Novel Coronavirus Pneumonia (Trial Version 7)", Chinese Medical Journal.

7. Tan, L., Wang, Q., Zhang, D., Ding, J., Huang, Q., Tang, Y.-Q., Wang, Q., \& Miao, H. (2020). Lymphopenia predicts disease severity of COVID19: a descriptive and predictive study, Signal transduction and targeted therapy 5, 1-3.

8. Sun, P., Lu, X., Xu, C., Sun, W., and Pan, B. (2020). Understanding of COVID- 19 based on current evidence, Journal of medical virology. 
9. Zhang, Y., Lin, D., Xiao, M., Wang, J., Wei, Y., Lei, Z., Zeng, Z., Li, L., Li, H., and Xiang, W. (2020). 2019-novel coronavirus infection in a three-month-old baby, Zhonghua er ke za zhi= Chinese journal of pediatrics 58, E006.

10. Association, P. B. o. H. M., \& Association, P. B. o. W. M. (2020). 湖北省儿童新型冠状病毒感染诊 疗建议 (试行第一版), 中国当代儿科杂志 $22,96-$ 99.

11. Henry, B. M., Lippi, G., \& Plebani, M. (2020). Laboratory abnormalities in children with novel coronavirus disease 2019, Clinical Chemistry and Laboratory Medicine (CCLM) 1.

12. Lu, X., Zhang, L., Du, H., Zhang, J., Li, Y., \& Qu, J. (2020). SARS-CoV-2 Infection in Children.[published online ahead of print, 2020 Mar 18], N Engl J Med 10.

13. Dong, Y., Mo, X., Hu, Y., Qi, X., Jiang, F., Jiang, Z., \& Tong, S. (2020). Epidemiological characteristics of 2143 pediatric patients with 2019 coronavirus disease in China, Pediatrics.

14. Huang, C., Wang, Y., Li, X., Ren, L., Zhao, J., Hu, Y., Zhang, L., Fan, G., Xu, J., \& Gu, X. (2020). Clinical features of patients infected with 2019 novel coronavirus in Wuhan, China, The lancet 395, 497-506.

15. Ki, M. (2020). Epidemiologic characteristics of early cases with 2019 novel coronavirus (2019$\mathrm{nCoV})$ disease in Korea, Epidemiology and health 42.

16. Yang, X., Yu, Y., Xu, J., Shu, H., Liu, H., Wu, Y., Zhang, L., Yu, Z., Fang, M., \& Yu, T. (2020) Clinical course and outcomes of critically ill patients with SARS-CoV-2 pneumonia in Wuhan, China: a single-centered, retrospective, observational study, The Lancet Respiratory Medicine.

17. Xu, X.-W., Wu, X.-X., Jiang, X.-G., Xu, K.-J., Ying, L.-J., Ma, C.-L., Li, S.-B., Wang, H.-Y., Zhang, S., \& Gao, H.-N. (2020) Clinical findings in a group of patients infected with the 2019 novel coronavirus (SARS-Cov-2) outside of Wuhan, China: retrospective case series, bmj 368.

18. Wang, D., Hu, B., Hu, C., Zhu, F., Liu, X., Zhang, J., Wang, B., Xiang, H., Cheng, Z., \& Xiong, Y. (2020) Clinical characteristics of 138 hospitalized patients with 2019 novel coronavirus-infected pneumonia in Wuhan, China, Jama 323, 10611069.

19. Xia, W., Shao, J., Guo, Y., Peng, X., Li, Z., \& Hu, D. (2020) Clinical and CT features in pediatric patients with COVID- 19 infection: Different points from adults, Pediatric pulmonology 55, 1169-1174.

20. Chen, N., Zhou, M., Dong, X., Qu, J., Gong, F., Han, Y., Qiu, Y., Wang, J., Liu, Y., \& Wei, Y. (2020) Epidemiological and clinical characteristics of 99 cases of 2019 novel coronavirus pneumonia in Wuhan, China: a descriptive study, The Lancet 395, 507-513.

21. Liu, W. J., Zhao, M., Liu, K., Xu, K., Wong, G., Tan, W., \& Gao, G. F. (2017) T-cell immunity of SARS-CoV: Implications for vaccine development against MERS-CoV, Antiviral research 137, 82-92.

22. Chu, H., Zhou, J., Wong, B. H.-Y., Li, C., Chan, J. F.-W., Cheng, Z.-S., Yang, D., Wang, D., Lee, A. C.-Y., \& Li, C. (2016). Middle East respiratory syndrome coronavirus efficiently infects human primary $\mathrm{T}$ lymphocytes and activates the extrinsic and intrinsic apoptosis pathways, The Journal of infectious diseases 213, 904-914.

23. Gu, J., Gong, E., Zhang, B., Zheng, J., Gao, Z., Zhong, Y., Zou, W., Zhan, J., Wang, S., \& Xie, Z. (2005) Multiple organ infection and the pathogenesis of SARS, The Journal of experimental medicine 202, 415-424.

24. Chen, F., Liu, Z., Zhang, F., Xiong, R., Chen, Y., Cheng, X., Wang, W., \& Ren, J. (2020) Frist case of severe childhood novel coronavirus pneumonia in China, Zhonghua er ke za zhi= Chinese journal of pediatrics 58, E005.

25. Lippi, G., \& Plebani, M. (2020) Procalcitonin in patients with severe coronavirus disease 2019 (COVID-19): A meta-analysis, Clinica Chimica Acta; International Journal of Clinical Chemistry.

26. Cai, J., Xu, J., Lin, D., Xu, L., Qu, Z., Zhang, Y., Zhang, H., Jia, R., Wang, X., \& Ge, Y. (2020) A Case Series of children with 2019 novel coronavirus infection: clinical and epidemiological features, Clinical Infectious Diseases.

27. Xiuli Ding, M., Geqing Xia, M., Zhi Geng, M., Wang, Z., \& Wang, L. A simple laboratory parameter facilitates early identification of COVID-19 patients.

28. Qu, R., Ling, Y., Zhang, Y. h. z., Wei, L. y., Chen, X., Li, X. m., Liu, X. y., Liu, H. M., Guo, Z., \& Ren, H. (2020) Platelet- to- lymphocyte ratio is associated with prognosis in patients with coronavirus disease- 19, Journal of medical virology.

29. Forget, P., Khalifa, C., Defour, J.-P., Latinne, D., Van Pel, M.-C., \& De Kock, M. (2017) What is the normal value of the neutrophil-to-lymphocyte ratio?, BMC research notes 10,12 .

30. Liu, J., Liu, Y., Xiang, P., Pu, L., Xiong, H., Li, C., Zhang, M., Tan, J., Xu, Y., \& Song, R. (2020) Neutrophil-to-lymphocyte ratio predicts severe illness patients with 2019 novel coronavirus in the early stage, MedRxiv.

31. Wenzhong, L., \& Hualan, L. COVID-19: Attacks the 1-Beta Chain of Hemoglobin and Captures the Porphyrin to Inhibit Human Heme Metabolism. ChemRxiv 2020, Preprint. https://doi. org/10.26434/chemrxiv 11938173, v6. 